Gynecology, Hasanuddin University, Makassar, Indonesia ${ }^{3}$ Division of Maternal Fetal Medicine, Department of Obstetrics and Gynecology, Hasanuddin University, Makassar, Indonesia

Objective: This study aims to assess adjuvant chemotherapy impacts on survival benefit at advanced stage epithelial ovarian cancer (EOC) treated with adjuvant chemotherapy.

Methods: A retrospective survival analysis between December 2018 to April 2021. Samples were all patients with advancedstage EOC treated with adjuvant chemotherapy. Characteristics of the study samples were assessed based on age, marital status, history of contraception, patient's outcome, International Federation of Gynaecology and Obstetrics (FIGO) staging, chemotherapy regimens, residual disease, and the number of toxicities that appeared. Advanced-stage was defined as stage IIB-IVB based on FIGO staging 2014. Chemotherapy regimens were paclitaxel-carboplatin and docetaxel-carboplatin. Residual disease was defined as residual disease $>1 \mathrm{~cm}$. We included patients with advanced-stage EOC who completing their adjuvant chemotherapy.

Results: Ninety-eight patients were identified. Factors that significantly influenced survival are FIGO staging, chemotherapy regimens, and residual disease ( $p=0.001)$. We also found a significant association number and type of toxicity to survival $(\mathrm{p}=0.001)$. Bone marrow toxicity $(26.6 \%)$, peripheral neuropathy (34.7\%), the combination of bone marrowgastrointestinal toxicities (29.6\%), and the combination of bone marrow-gastrointestinal toxicities-nephropathy (7.1\%) were identified as side effects that appeared after completing adjuvant chemotherapy. The shortest survival was found in the combination of bone marrow-gastrointestinal toxicities and nephropathy (median=12.5 months).

Conclusion: The combination of bone marrow toxicity, gastrointestinal tract toxicity, and nephropathy is the most lethal complication which decreased the length of survival after completing adjuvant chemotherapy in advanced-stage EOC.

Poster (019)

Basic/Translational Science

https://doi.org/10.3802/jgo.2021.32.S1.019

\section{Targeting nuclear F-actin to circumvent ovarian cancer chemotherapy resistance}

\section{Wei Wu, Xiaoxia Xing, Rongyu Zang, Yiyan Zheng}

Department of Gynecologic, Oncology, Zhongshan Hospital, Fudan University, Shanghai, China (yiyanzheng@fudan.edu.cn)

Objective: Despite an excellent initial response to chemotherapy, a large proportion of ovarian cancer patients are faced with tumour relapse within 2 years. The chemotherapy drugs, cisplatin or carboplatin, induce DNA damage and results in cell apoptosis in ovarian cancer. The underlying mechanisms of how ovarian cancer cells acquire resistance to chemotherapy remain obscure. It is widely accepted that actin filament (F-actin) is one of the main cytoskeleton that is mainly organized in the cytoplasm, and it is involved in key physiological processes, e.g. cell division, cell migration and intracellular trafficking. However, this has been challenged by recent studies showing that F-actin and globular actin exist in the nuclei of somatic cells, and are involved in chromatin remodeling and gene transcription regulation. Notably, 2 pioneering studies have claimed that nuclear F-actin is assembled to accelerate DNA repair in fly and mouse cells. Our preliminary data reveals that nuclear F-actin organization occurs in ovarian cancer cells, and we have found that cells with these structures are less sensitive to carboplatin treatment. Therefore, we hypothesize that nuclear F-actin assembly may have a critical role in chemotherapy resistance in ovarian cancer cells. To date, the clinical significance of nuclear F-actin has not been investigated and our study may provide insight into this exciting link.

Methods: Ovarian cancer cell lines stably expressing nuclear actin-chromobody-citrine probe were used to investigate nuclear F-actin structure, in combination with spinning disc confocal microscopy and super resolution stimulated emission depletion microcopy. The nuclear F-actin structures were further evaluated in ovarian cancer patient tumour samples using phalloidin staining, a toxin which specifically binds to F-actin. Additionally, IF staining using cell apoptosis marker antibody (anti-caspase 3) and long-term colony formation assays were conducted to investigate the chemosensitivity of ovarian cancer cells with nuclear F-actin. Moreover, we performed kinome screening using kinome small interfering RNA library and small molecular inhibitors library (TargetMol library) in combination with High-Content Screening system to unravel the underlying molecular pathways which may potentially be involved in regulating nuclear F-actin polymerization in ovarian cancer cells.

Results: We generated ovarian cancer cell lines stably expressing nuclear actin chromobody and found out that nuclear F-actin were polymerized in ovarian cancer cells, which was further confirmed using phalloidin staining. To investigate the clinical significance of nuclear F-actin in the context of ovarian cancer, we conducted F-actin staining using phalloidin in tumour tissues from individual ovarian cancer patients. It was confirmed that nuclear F-actin structures were present in ovarian tumour cells from patient samples. Moreover, cells with nuclear F-actin had less rH2Ax foci under the treatment of platinum drugs, indicating that nuclear F-actin might play critical roles in DNA repair in ovarian cancer cells. Importantly, ovarian cancer cells with the nuclear F-actin 
phenotype had better survival to chemotherapy treatment. At the molecular level, we found out that the mitogenactivated protein kinase, phosphoinosytol-3 phosphate/AKT pathways and ABL1 kinase are involved in nuclear F-actin polymerization. Further investigation revealed that ABL1 may phosphorylate Wiskott-Aldrich syndrome protein to activate the key actin nucleation factor Arp2/3 complex to promote nuclear F-actin polymerization in ovarian cancer cells treated with chemotherapy drugs carboplatin or cisplatin. Our preliminary data suggests a potential target in combination with chemotherapy for ovarian cancer treatment, and this necessitates further evaluation by recruiting preclinical models in future studies.

Conclusion: Here, we reveal that ovarian cancer cells with nuclear F-actin undergo less DNA damage under platinum treatment, suggesting that nuclear F-actin may mediate chemotherapy resistance in the context of ovarian cancer. Moreover, we unravel the underlying kinase pathway involved in nuclear F-actin polymerization in ovarian cancer cells, potentially paving the way for a novel strategy of targeting potential kinases in combination with well-established chemotherapy regimens in order to circumvent chemotherapy resistance.

Poster (020)

Imaging in Gynecologic Cancer

https://doi.org/10.3802/jgo.2021.32.\$1.020

\section{The diagnostics of epithelial ovarian cancer: focus on apparent diffusion coefficient map on magnetic resonance imaging using machine learning}

\author{
Heekyoung Song, ' Jae Yeon Woo,' Seongeun Bak, ${ }^{1}$ \\ Imhyeon Kim, ${ }^{1}$ Youn Jin Choi, ${ }^{1}$ Sung Eun Rha, ${ }^{2}$ Seo Yeon Youn, ${ }^{2}$ \\ Shin Ah Oh, ${ }^{3}$ Sung Jong Lee ${ }^{1, *}$ \\ 'Department of Obstetrics and Gynecology, Seoul St. Mary's Hospital, \\ College of Medicine, The Catholic University of Korea, Seoul, Korea \\ (orlando@catholic.ac.kr) \\ 2Department of Radiology, Seoul St. Mary's Hospital, College of Medicine, The \\ Catholic University of Korea, Seoul, Korea 3NAVER Clova AI, Seongnam, Korea
}

Objective: To compare radiologic features including mean apparent diffusion coefficient (ADC) value of uterus magnetic resonance imaging (MRI) among the histology types of epithelial ovarian cancer (EOC).

Methods: This retrospective single center study included patients underwent preoperative uterus MRI and pathologically diagnosed as EOC. On MRI, the proper image of the axial ADC map that contained the largest area of the solid portion was selected, and the region of interest (ROI) of the solid portion were manually drawn by a genecologist and a radiologist with consensus. The mean $\mathrm{ADC}$ value (ADCmean) of solid portion was derived from ROI. The ADCmean in each histology was compared using MannWhitney $\mathrm{U}$ test. The 3 kinds of machine learning (ML) algorithms also used to evaluate several values included ADCmean (Random Forest, Gradient Boosting Machine, and XGBoost).

Results: Two hundred patients were finally included with 97 type I patients (low-grade serous carcinoma, $\mathrm{n}=8$; mucinous carcinoma $[\mathrm{MC}], \mathrm{n}=33$; endometroid carcinoma [EC], $\mathrm{n}=21$; clear cell carcinoma [CCC], $\mathrm{n}=35$ ) and 103 type II patients (high-grade serous carcinoma [HGSC], $\mathrm{n}=103$ ). The ADCmean significantly differ between type I and II EOC $(\mathrm{p}<0.001)$. In detail, median ADCmean of HGSC was $1.06 \times 10^{-3} \mathrm{~s} / \mathrm{m}^{2}$ the lowest among all types, and significantly distinguished from MC, EC, and CCC ( $\mathrm{p} \leq 0.001)$. Among 3 types of ML, Gradient Boosting Machine noted the highest accuracy to distinguish type I/II EOC (accuracy: 0.91), XGBoost conducted the highest value in 5 histology of EOC (accuracy: 0.68).

Conclusion: The ADCmean is useful tool to evaluate the histologic type of EOC in preoperative MRI.

Poster (O21)

Epithelial Ovarian Cancer including Borderline Tumor

https://doi.org/10.3802/jgo.2021.32.S1.021

\section{Toxicity profile of olaparib maintenance monotherapy for Japanese platinum-sensitive relapsed ovarian, fallopian tube and primary peritoneal cancer patients}

\section{Tomoko Yoshihama, Tatsuyuki Chiyoda, "Yuka Kuroda, Yusuke Kobayashi, Wataru Yamagami, Daisuke Aoki}

Department of Obstetrics and Gynecology, Keio University School of Medicine, Tokyo, Japan (chiyoda@keio.jp)

Objective: This study aims to investigate the toxicity profile of olaparib maintenance therapy for Japanese population.

Methods: Fifty-two Japanese patients with platinum-sensitive relapsed ovarian, fallopian tube and primary peritoneal cancer who started olaparib maintenance monotherapy following platinum-based chemotherapy from May 2018 to June 2021 at our hospital were included. The information about the adverse effect were collected retrospectively from the medical records. This study was approved by the ethics committee of Keio University, School of Medicine.

Results: The median age was 58 years old (33-80 years), and the median progression-free survival was 17.1 months $(95 \%$ confidence interval=10.6-23.5). The number of previous platinum-based regimens was 2 in 35 patients (67.3\%), 3 in 8 patients $(15.4 \%)$ and $\geq 4$ in 9 patients $(17.3 \%)$. Twenty-nine 